\title{
OPEN Influence of InP coupling cavity on Fano resonance of sub wavelength MIM waveguide
}

\author{
Shihao Ban ${ }^{1}$, Huangqing Liu ${ }^{1 \bowtie}$, Shifang Xiao ${ }^{1}$, Jingjing Mao ${ }^{2 \bowtie}$ \& Jie Luo ${ }^{3}$ \\ In this paper, influence of InP coupling cavity on Fano resonance of sub wavelength MIM waveguide \\ was studied by FDTD. It was observed that the resonant wavelengths of mode $m_{j}(j=1,2,3)$ were \\ closely related with the height $\mathrm{H}_{2}$ of InP coupling cavity. In addition, before and after the addition of \\ air cavity, the relative farfield intensities I was a function of height $H_{2}$. Therefore, InP as discrete state \\ could be used as the filling dielectrics of Fano resonance in the MIM waveguide.
}

Due to its attractive features and extensive applications, the metal-insulator-metal (MIM) waveguide ${ }^{1-5}$ were very popular with researchers. During the process of light propagation in the nano-photonic circuits, MIM waveguides could reduce energy loss. The MIM waveguide with coupling cavity could change its filtering performance ${ }^{6-9}$, such as Fano resonance. The most common structures with the filters were MIM waveguides with a rectangular cavity $^{10-14}$. Silicon was used as filter dielectrics in the coupling cavity ${ }^{13,14}$. Due to relatively large dielectric constant and high-order resonance about $1700 \mathrm{~nm}$ in the near-infrared region, silicon was usually regarded as discrete state of Fano resonance ${ }^{13-18}$. In the study, obvious Fano resonance could be observed when InP was chose as the dielectrics in the coupling cavity of MIM waveguide. Namely, InP could also be used as discrete state of Fano resonance. In this paper, In $\mathrm{P}^{19-25}$ would be used as the filling dielectrics in the MIM waveguide cavity and the influence of InP coupling cavity on Fano resonance of sub wavelength MIM waveguide (Ag-Air-Ag) was explored on the basis of the literatures $22,25,26$.

\section{Results}

The sketch of the waveguide structure designed was shown in Fig. 1. In the figure, the distance $w$ of the main waveguide cavity with the transparent dielectrics $(n=1.0)$ was set as $50 \mathrm{~nm}$ which was far less than the wavelength $\lambda$ of incident wave so that SPPs propagation mode could be excited in MIM waveguide structure because the number of SPP modes was closely related to the distance along the wave propagation direction ${ }^{27}$. In addition, the width $\boldsymbol{L}$ of the coupled cavities was also set as $50 \mathrm{~nm}$. Our simulation results indicated that Fano resonance was independent of the horizontal distance between the two cavities and dependent on the height $\boldsymbol{H}_{1}\left(\boldsymbol{H}_{2}\right)$. Additionally, the relative far-field intensity $I$ was defined as the area under the far-field curve and was proportional to the height $h$ of the curve in this paper.

Figure 2a showed the transmission spectra under different InP structures without air cavity $\mathbf{A}$. It could be observed that the resonance peaks of mode $\mathrm{m}_{\mathrm{j}}(\mathrm{j}=1,2,3)$ redshifted with the increase of $H_{2}$ from 240 to $350 \mathrm{~nm}$. And that the resonant wavelengths were a function of the height $H_{2}$. Take $H_{2}=350 \mathrm{~nm}$ as an example, it could be observed three resonance valleys at about $998 \mathrm{~nm}, 1302 \mathrm{~nm}$ and $2079 \mathrm{~nm}$, respectively. The resonance valleys at $1302 \mathrm{~nm}$ and $2079 \mathrm{~nm}$ were considered to be first and second order resonance modes, shown in Fig. 2c,d. However, the resonance valley at $998 \mathrm{~nm}$ was regarded as third-order resonance (seen in Fig. 2a,b), which was independent of the height $\boldsymbol{H}_{1}$ and closely related to the height $H_{2}$ according to our simulations. In other words, the resonance mode in Fig. $2 \mathrm{a}$ could directly be confirmed by the distribution of magnetic field in Fig. $2 \mathrm{~b}-\mathrm{d}$.

When the zero order dark mode from the Air cavity and $j$-order bright modes $(j=1,2$ and 3$)$ from the InP cavity were superimposed, some Fano resonance of the modes $m_{j}(j=1,2$ and 3$)$ could be obtained in Fig. 3a, which showed the transmission spectra of different InP-Air structures. In the case of only air cavity, the transmission spectrum was very wide passband, which covered the resonance wavelengths of the modes $m_{j}(j=1$, 2 and 3 ) and whose central wavelength was lied at about $1710 \mathrm{~nm}$. For the mode $\mathrm{m}_{1}$, more obvious Fano resonance was observed when $\mathrm{H}_{2}=240 \mathrm{~nm}$ and $260 \mathrm{~nm}$. However, the most obvious Fano resonance came from the mode $\mathrm{m}_{2}$ whose resonance valley shifted to the long wavelength with the $\mathrm{H}_{2}$ increase. According to the main

\footnotetext{
${ }^{1}$ School of Physics and Electronics, Hunan University, Changsha 410082, People's Republic of China. ${ }^{2}$ Huaihua Normal College, Huaihua 418008, People's Republic of China. ${ }^{3}$ School of Material Science and Engineering, Central South University of Forestry and Technology, Changsha 410004, Hunan, People's Republic of China. ${ }^{\boxplus e m a i l:}$ Ihq468@sohu.com; mjj6937213@163.com
} 


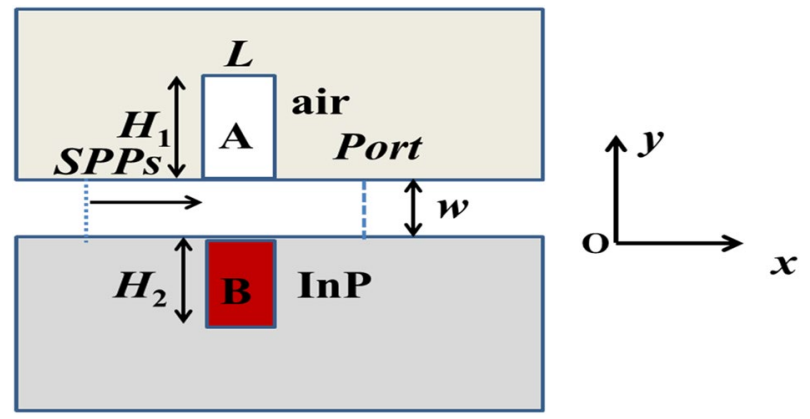

Figure 1. Research scheme and design schematic of $2 \mathrm{D}$ simulation. The capital letters $\mathrm{A}$ and $\mathrm{B}$ denote air and InP coupled cavities, respectively. The structural parameters were $L=w=50 \mathrm{~nm}$.
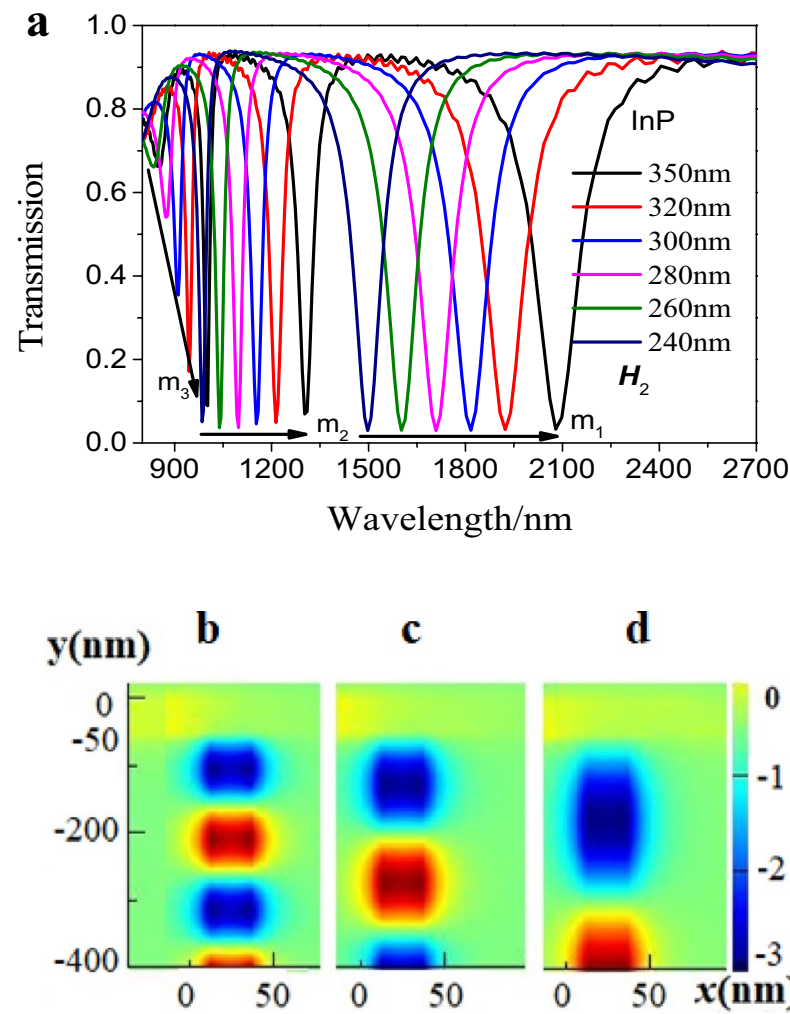

c

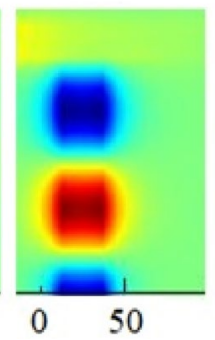

d

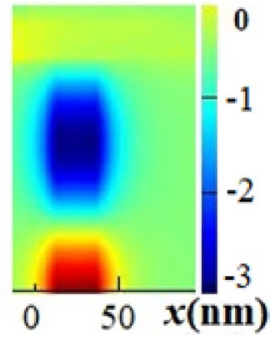

Figure 2. (a) The relation between Fano resonance and the height $H_{2}$. (b-d) Magnetic field distribution of different modes at different resonance wavelengths $998 \mathrm{~nm}, 1302 \mathrm{~nm}$ and $2079 \mathrm{~nm}$, respectively. Red and blue represent two different vibrations. The structural parameter was $\mathrm{H}_{2}=350 \mathrm{~nm}$.

resonance peak ${ }^{22-23}$ of the mode $\mathrm{m}_{2}$ in the Fig. 3a, the quality factor (QF) and the extinction ratio (ER) could be calculated ${ }^{19,20,23,26}$. The quality factor $Q$ was defined as the ratio of the resonance wavelength $\lambda_{0}$ and the full width $\Delta \lambda$ between the peak and the antipeak of the transmission $\left(\mathrm{Q}=\lambda_{0 /} \Delta \lambda\right)$. The extinction ratio (Ext) was ten times the logarithm of the ratio of the maximum transmission light intensity $P$ to the minimum transmitted light intensity $P_{0}$, namely, Ext $=10 \lg \left(P / P_{0}\right)$. A high-quality factor meant lower light energy loss in a resonant cavity. Moreover, the lager extinction ratio was implied the better quality of the resonator. For the different InP-Air structures with the length $\mathrm{H}_{2}$ from 240 to $350 \mathrm{~nm}(240 \mathrm{~nm}, 260 \mathrm{~nm}, 280 \mathrm{~nm}, 300 \mathrm{~nm}, 320 \mathrm{~nm}$ and $350 \mathrm{~nm})$, the quality factor QFs calculated were about $44,30,29,28,34$ and 37 , respectively. The ERs obtained were around $13,18,15,14,31$ and $12 \mathrm{~dB}$, respectively. These results showed that the QFs and the ERs were a function of the height $\boldsymbol{H}_{2}$. There was lower light energy loss in a resonant cavity and better quality of the resonator for the length $\boldsymbol{H}_{2}=240 \mathrm{~nm}$ and $320 \mathrm{~nm}$. In other words, when the height $\boldsymbol{H}_{2}$ was not higher than $240 \mathrm{~nm}$ and not less than $320 \mathrm{~nm}$, the waveguide structure with InP-Air cavities had good filtering performance. In addition, it was obtained that the addition of air cavity did not change the resonant position which was dependent on structure sizes of the cavity InP. Take $\mathrm{H}_{2}=350 \mathrm{~nm}$ as an example, for the three resonance valleys at $998 \mathrm{~nm}, 1302 \mathrm{~nm}$ and $2079 \mathrm{~nm}$, it was observed the magnetic field distribution did not change before and after the addition of air 

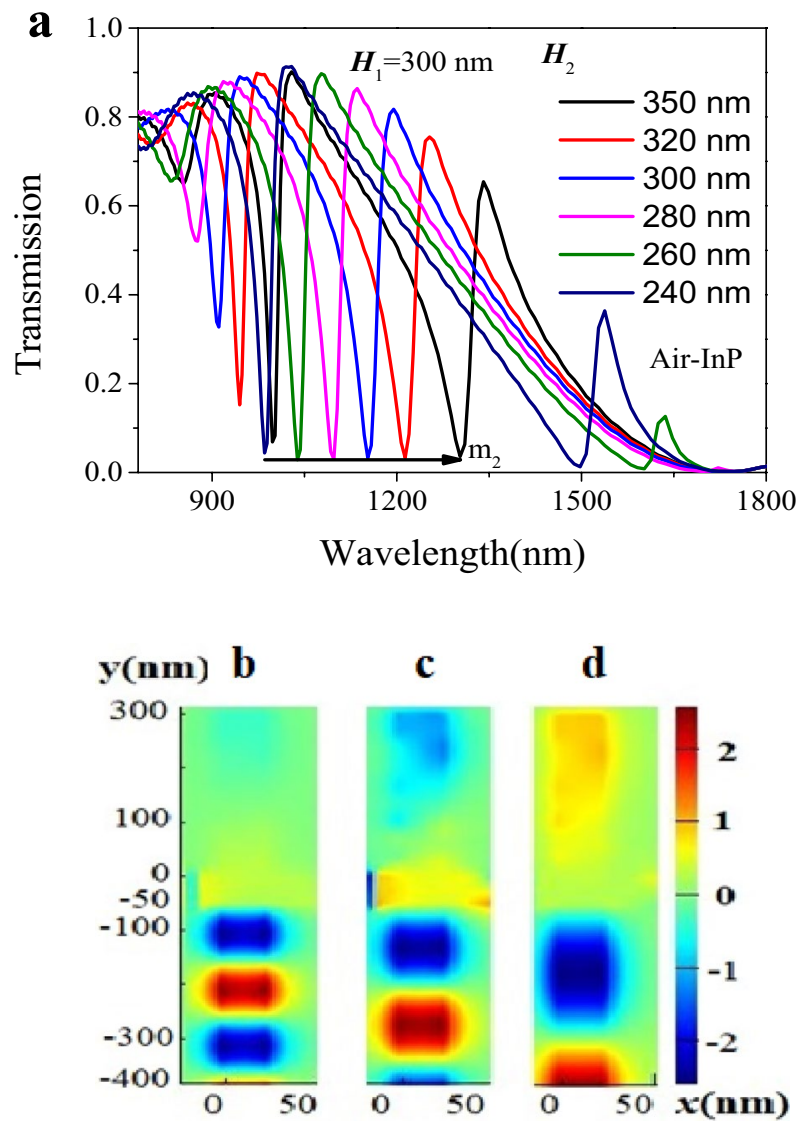

Figure 3. (a) The relationship between Fano resonance and the height $H_{2}$ in the range from 780 to $1800 \mathrm{~nm}$. (b-d) Magnetic field distribution of different modes at different resonance wavelengths $998 \mathrm{~nm}, 1302 \mathrm{~nm}$ and $2079 \mathrm{~nm}$, respectively. Red and blue represent two different vibrations. The structural parameter was $H_{1}=300 \mathrm{~nm}$.

cavity. Additionally, it was obtained that the magnetic field distribution of the zero-order resonance in the air cavity, as shown in Fig. 3b-d.

Before the addition of air cavity, under $1302.87 \mathrm{~nm}$ monitoring, the relative farfield intensities $I$ varied with the height $\boldsymbol{H}_{2}$ from 240 to $350 \mathrm{~nm}$ and was a function of height $\boldsymbol{H}_{2}$, namely, $I=I\left(\boldsymbol{H}_{2}\right)$, as shown in Fig. $4 \mathrm{a}$. The shape of the curve was Guassian Spot with periodic structure, whose symmetric center was located at about $\theta=-2.2^{\circ}$, as shown in the black dash. The relative farfield intensity $I$ gradually decreased with the height $\boldsymbol{H}_{2}$ from 260 to $350 \mathrm{~nm}$. The maximum relative intensity $I(260)$ was about three times that of the minimum $I(350)$, $I(260)=3 I(350)$. After the addition of air cavity, it was observed that the relative farfield intensities $I$ was also a function of height $\boldsymbol{H}_{2}$, as shown in Fig. $4 \mathrm{~b}$. The symmetric center of the curve with periodic structure was located at about $\theta=0^{\circ}$, as seen in the black dash. The relative farfield intensity $I$ gradually increased with the height $\boldsymbol{H}_{2}$ from 240 to $320 \mathrm{~nm}$. The maximum relative intensity $I(320)$ was about four times that of the minimum $I(350)$, namely, $I(320)=4 I(350)$. Therefore, the change of structural parameters could be obtained according to the change of far-field relative intensity ${ }^{20}$.

\section{Discussion}

Influence of InP coupling cavity on Fano resonance of sub wavelength MIM waveguide was studied in this paper. Some novel results were obtained. For different InP structures without air cavity, it was observed the resonance peaks of mode $m_{j}(j=1,2$ and 3$)$ redshifted with $H_{2}$ increase and the resonant wavelengths of mode $m_{j}(j=1,2$ and 3 ) were a function of the height $\mathrm{H}_{2}$. For the different InP-Air structures with the length $\mathrm{H}_{2}$ from 240 to $350 \mathrm{~nm}$, the resonance valley of the mode $\mathrm{m}_{2}$ shifted to the long wavelength. In addition, before and after the addition of air cavity, the relative farfield intensities $I$ was a function of the height $H_{2}$. Therefore, InP as discrete state of Fano resonance could be used as the filling dielectrics of Fano resonance in the MIM waveguide.

\section{Methods}

Numerical simulations. In the letter, the MIM plasmonic waveguide coupled with InP cavity was investigated using the finite-difference time-domain (FDTD, Lumerical Computational Solutions Incorporation) with a perfectly matched layer absorbing boundary condition. A plane wave with the electric field parallel to the $x$ axis illuminates normally the periodic structure. The grid sizes in the $x$ and $y$ directions were $2 \mathrm{~nm}$. 


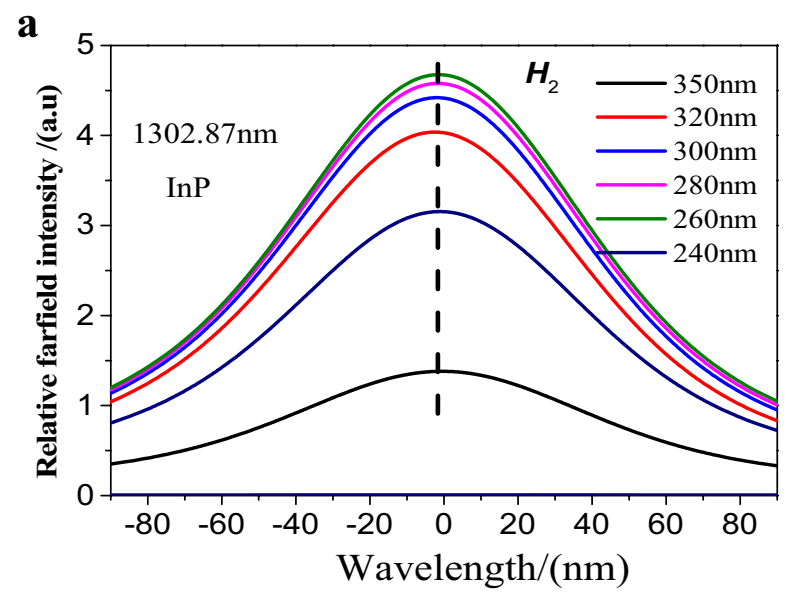

b

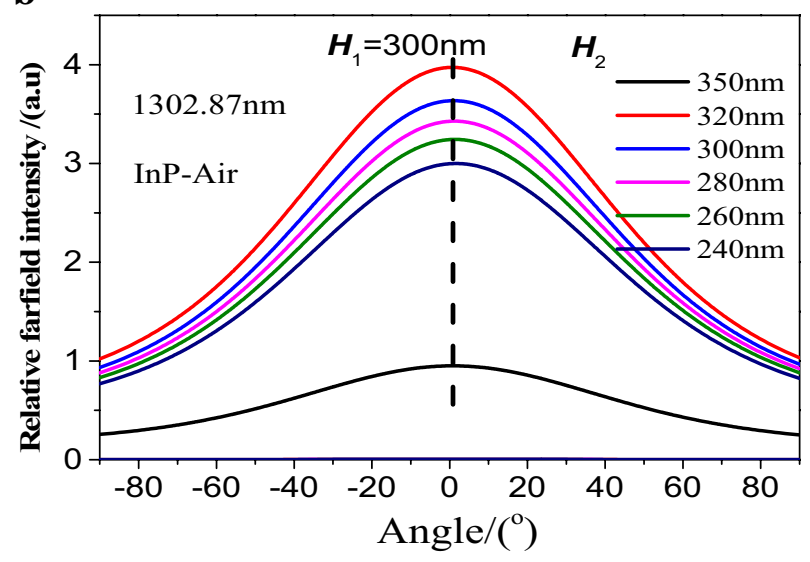

Figure 4. Farfield with vavious angle from $-90^{\circ}$ to $90^{\circ}$ and changeable height $H_{2}$ from 240 to $350 \mathrm{~nm}$ before (a) and after (b) the addition of air cavity under $1302.87 \mathrm{~nm}$ monitoring.

Received: 13 March 2021; Accepted: 12 May 2021

Published online: 02 June 2021

\section{References}

1. Törmä, P. \& Barnes, W. L. Strong coupling between surface plasmon polaritons and emitters: A review. Rep. Prog. Phys. 78, 013901. https://doi.org/10.1088/0034-4885/78/1/013901 (2015).

2. Abdol, S. O. et al. Tunable surface plasmon polaritons in a Weyl semimetal waveguide. J. Phys. Condens. Matter 31, 335002. https:// doi.org/10.1088/1361-648X/ab217f (2019).

3. Lošić, ŽB. Surface plasmons in Weyl semimetals. J. Phys. Condens. Matter 30, 365003. https://doi.org/10.1088/1361-648X/aad6f5 (2018).

4. Saleh, N. M. \& Aziz, A. A. Simulation of surface plasmon resonance on different size of a single gold nanoparticle. J. Phys. Conf. Ser. 1083, 012041. https://doi.org/10.1088/1742-6596/1083/1/012041 (2018).

5. Cao, E. et al. Exciton-plasmon hybrids for surface catalysis detected by SERS. Nanotechnology 29, 372001. https://doi.org/10.1088/ 1361-6528/aacec4 (2018).

6. Melentev, G. A. et al. Surface plasmon-phonon polaritons in GaAs. J. Phys. Conf. Ser. 917, 062038. https://doi.org/10.1088/17426596/917/6/062038 (2017).

7. Kim, Y. et al. Demonstration of integrated polarization rotator based on an asymmetric silicon waveguide with a trench. J. Opt. 18, 095801. https://doi.org/10.1088/2040-8978/18/9/095801 (2016).

8. Ghosh, S. et al. Transverse localization of light in 1D disordered waveguide lattices in the presence of a photonic bandgap. Laser Phys. 24, 045001. https://doi.org/10.1088/1054-660X/24/4/045001 (2014).

9. Piccione, B. et al. Tailoring light-matter coupling in semiconductor and hybrid-plasmonic nanowires. Rep. Prog. Phys. 77, 086401. https://doi.org/10.1088/0034-4885/77/8/086401 (2014).

10. Wen, K. et al. Wavelength demultiplexing structure based on a plasmonic metal-insulator-metal waveguide. J. Opt. 14, 075001. https://doi.org/10.1088/2040-8978/14/7/075001 (2012).

11. Sheng, Y. et al. Fabrication of lateral electrodes on semiconductor nanowires through structurally matched insulation for functional optoelectronics. Nanotechnology 24, 025204. https://doi.org/10.1088/0957-4484/24/2/025204 (2013).

12. Yun, B., Hu, G. \& Wei, C. J. Plasmon induced transparency in metal-insulator-metal waveguide by a stub coupled with F-P resonator. Mater. Res. Express 1, 036201. https://doi.org/10.1088/2053-1591/1/3/036201 (2014).

13. Zhong, X. \& Li, Z. Plasmon enhanced light amplification in metal-insulator-metal waveguides with gain. J. Opt. 14, 055002. https://doi.org/10.1088/2040-8978/14/5/055002 (2012).

14. Wang, Y. \& Yan, X. Mode conversion in metal-insulator-metal waveguide with a shifted cavity. Jpn. J. Appl. Phys. 57, 010303 (2018).

15. Zheng, G., Su, W. \& Chen, Y. Band-stop filters based on a coupled circular ring metal-insulator-metal resonator containing nonlinear material. J. Opt. 14, 055001. https://doi.org/10.1088/2040-8978/14/5/055001 (2012). 
16. Nurmohammadi, T. et al. Ultra-fast all-optical plasmon induced transparency in a metal-insulator-metal waveguide containing two Kerr nonlinear ring resonators. J. Opt. 20, 055504. https://doi.org/10.1088/2040-8986/aab882 (2018).

17. Yun, B., Hu, G. \& Cui, Y. Theoretical analysis of a nanoscale plasmonic filter based on a rectangular metal-insulator-metal waveguide. J. Phys. D Appl. Phys. 43, 385102 (2010).

18. Li, S., Liu, H. \& Liu, L. Effect of silver film thickness on the surface plasma resonance in the rectangular Ag-Si-SiO 2 cavity. J. Phys. Commun. 2, 055024. https://doi.org/10.1088/2399-6528/aac52e (2018).

19. $\mathrm{Li}$, S. et al. Investigation of surface plasmon resonance in the rectangular cavity of $\mathrm{Ag}-\mathrm{Si}-\mathrm{SiO}_{2}$. Plasmonics 13(6), 2313. https:// doi.org/10.1007/s11468-018-0754-y (2018).

20. Li, S. et al. Farfield under small scattering angle in the rectangular Ag-Si-SiO ${ }_{2}$ cavity. Plasmonics 14, 1385. https://doi.org/10.1007/ s11468-019-00928-7 (2019).

21. Ghorbani, S., Dashti, M. A. \& Jabbar, M. Plasmonic nano-sensor based on metal-dielectric-metal waveguide with the octagonal cavity ring. Laser Phys. 28, 066208. https://doi.org/10.1088/1555-6611/aab463 (2018).

22. Mao, J. et al. Numerical analysis of near-infrared plasmonic filter with high figure of merit based on Fano resonance. Appl. Phys. Express 10, 082201. https://doi.org/10.7567/APEX.10.082201 (2017).

23. Zhang, S., Li, G. \& Chen, Y. Pronounced fano resonance in single gold split nanodisks with $15 \mathrm{~nm}$ split gaps for intensive second harmonic generation. ACS Nano 10, 11105-11114. https://doi.org/10.1021/acsnano.6b05979 (2016).

24. Ren, D. K., Farrell, C. A., Diana, L. \& Huffaker, L. Axial InAs(Sb) inserts in selective-area InAsP nanowires on InP for optoelectronics beyond $2.5 \mu \mathrm{m}$. Opt. Mater. Express 8(4), 1075-1081. https://doi.org/10.1364/OME.8.001075 (2018).

25. Bekele, D. A. et al. Optical time domain demultiplexing using fano resonance in InP photonic crystals. In The European Conference on Lasers and Electro-Optics, Paper CK_1_5 (2017).

26. Li, S. et al. Fano resonance of air-Si waveguide cavity. Plasma Res. Express 2(4), 045005. https://doi.org/10.1088/2516-1067/abc467 (2020).

27. Li, H. et al. Graphene-based mid-infrared, tunable, electrically controlled plasmonic filter. Appl. Phys. Express 7, 024301. https:// doi.org/10.7567/APEX.7.024301 (2014).

\section{Acknowledgements}

This work was supported by the National Natural Science Foundation of China (Grant No. 51571088) and the Natural Science Foundation of Hunan Province (No. 2019JJ40535).

\section{Author contributions}

J.J.M. designed the Fig. 1. S.H.B. made a simulation according to the design and processed the pictures of the simulated magnetic field (Figs. $2 b-d, 3 b-d$ ) by using drawing software. H.Q.L made the figures (Figs. 2a, 3a, $4 \mathrm{a}, \mathrm{b})$ by using OriginPro 9.0 according to the simulated data and participated in the analysis and interpretation of all figures. All the authors participated in the writing of the article. S.F.X. and J.L. provided financial support.

\section{Competing interests}

The authors declare no competing interests.

\section{Additional information}

Correspondence and requests for materials should be addressed to H.L. or J.M.

Reprints and permissions information is available at www.nature.com/reprints.

Publisher's note Springer Nature remains neutral with regard to jurisdictional claims in published maps and institutional affiliations.

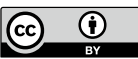

Open Access This article is licensed under a Creative Commons Attribution 4.0 International License, which permits use, sharing, adaptation, distribution and reproduction in any medium or format, as long as you give appropriate credit to the original author(s) and the source, provide a link to the Creative Commons licence, and indicate if changes were made. The images or other third party material in this article are included in the article's Creative Commons licence, unless indicated otherwise in a credit line to the material. If material is not included in the article's Creative Commons licence and your intended use is not permitted by statutory regulation or exceeds the permitted use, you will need to obtain permission directly from the copyright holder. To view a copy of this licence, visit http://creativecommons.org/licenses/by/4.0/.

(c) The Author(s) 2021 